\title{
ALTERNATIVE APPROACH TO MULTIVESSEI CORONARY DISEASE WITH INTEGRATED CORONARY REVASCULARIZATION
}

Marco Zenati, MD

Howard A. Cohen, MD

Bartley P. Griffith, MD
Objective: Integrated coronary revascularization combines minimally invasive coronary artery bypass grafting (MICABG) with left internal thoracic artery-left anterior descending artery grafting and percutaneous coronary intervention. We hypothesized that integrated coronary revascularization could result in successful revascularization in suitable patients with multivessel coronary artery disease. Methods: Between September 1996 and January 1998, 31 consecutive patients underwent integrated coronary revascularization. Twenty-two were male; mean age was 69 years (46-86 years) and $42 \%$ were older than 75 years. Eight patients $(26 \%)$ had a Parsonnet score greater than $20 \%$. Left ventricular ejection fraction was $46.3 \% \pm 12 \%$; 6 patients $(19 \%)$ had a left ventricular ejection fraction less than $35 \%$. Results: The anastomosis time for MICABG with the internal thoracic artery was $14.6 \pm 5.2$ minutes and the operating time was $105 \pm 20$ minutes; 28 patients $(90 \%)$ were extubated in the operating room. The internal thoracic artery anastomosis was patent in all 31 patients $(100 \%)$. Percutaneous coronary intervention was performed before MICABG in 2 patients $(7 \%)$, on the same day of MICABG in 16 patients (52\%), on postoperative day 1 in 3 patients $(9 \%)$, and on postoperative days 2 to 4 in 10 patients (32\%). Postprocedure length of stay in the hospital was $2.7 \pm 1.0$ days and 13 patients $(42 \%)$ were discharged home on postoperative day 1 or 2 . Three patients $(9.6 \%)$ required repeat target vessel revascularization in the distribution of the previous percutaneous coronary intervention. All patients are alive without angina at a follow-up of $10.8 \pm 3.8$ months. Conclusion: Our early results demonstrate that integrated coronary revascularization can be performed safely and effectively. Long-term results will be available from a prospective randomized trial now underway to compare integrated coronary revascularization with coronary artery bypass grafting for multivessel coronary artery disease. (J Thorac Cardiovasc Surg 1999;117:439-46)
T he search for the optimal strategy for myocardial revascularization is still in progress, 30 years after the first coronary artery bypass graft $(\mathrm{CABG})$ operation

From the Divisions of Cardiothoracic Surgery and Cardiology, University of Pittsburgh Medical Center, Pittsburgh, Pa.

Read at the Twenty-fourth Annual Meeting of The Western Thoracic Surgical Association, Whistler, British Columbia, June 24-27, 1998.

Received for publication July 15, 1998; revisions requested Oct 6, 1998; revisions received Nov 9, 1998; accepted for publication Nov 19, 1998.

Address for reprints: Marco Zenati, MD, Division of Cardiothoracic Surgery, 200 Lothrop St, Suite C-700, Pittsburgh, PA 15213-2582. Copyright (C) 1999 by Mosby, Inc.

$0022-5223 / 99 \$ 8.00+0 \quad \mathbf{1 2 / 6 / 9 6 1 9 8}$ and more than 20 years after the first percutaneous coronary angioplasty. The ideal revascularization strategy should be safe and effective, provide the best immediate and long-term result, be associated with minimal morbidity and invasiveness, be inexpensive, and require a short hospitalization.

In the past few years a rapid expansion of the available options for myocardial revascularization has occurred both in the interventional cardiology and in the cardiac surgery field (Table I); as a result, the gap between percutaneous and surgical coronary revascularization is narrowing, as percutaneous techniques move from "plain old balloon angioplasty" to more invasive and costly techniques ${ }^{1}$ and surgery is moving toward less invasive and less expensive approaches. ${ }^{2}$ 
Table I. Myocardial revascularization strategies

\begin{tabular}{l} 
Percutaneous coronary intervention \\
Percutaneous transluminal coronary angioplasty \\
Stenting \\
Atherectomy \\
Laser angioplasty \\
Percutaneous transmyocardial laser revascularization \\
Combination of the above \\
Surgical revascularization \\
CABG \\
MICABG \\
Transmyocardial laser revascularization \\
Integrated coronary revascularization (MICABG + PTCA/stent) \\
\hline
\end{tabular}

The successful and cost-effective application of minimally invasive CABG (MICABG) $)^{3,4}$ has created the basis for a new approach to multivessel coronary artery disease (CAD) by combining the minimally invasive surgical revascularization of the left anterior descending coronary artery (LAD) using the left internal thoracic artery (LITA) with percutaneous coronary intervention of the circumflex or right coronary artery. ${ }^{5,6}$

We hypothesized that integrated coronary revascularization, defined as the combination of MICABG and percutaneous coronary intervention, could provide the best revascularization strategy for patients with multivessel CAD involving the LAD, resulting in a safe and efficacious treatment with the best possible short- and long-term results at low cost and short hospital stay. We describe in this report the results of a feasibility study of integrated coronary revascularization at a single center.

\section{Methods}

The first MICABG was performed at the University of Pittsburgh in June 1995. Through May 1998, 189 procedures have been performed; our angiographic patency for ITA anastomosis is $97.7 \%$ (84/86 consecutive LITA angiographies from September 1996 to September 1997).

Since September 1996, our Minimally Invasive Cardiac Surgery Program joined forces with the Interventional Cardiology group to evaluate the feasibility of integrated coronary revascularization and explore its potential scope of applicability.

A pilot study of 31 patients that met inclusion criteria for integrated coronary revascularization (detailed in the Patient selection section) was performed from September 1996 to January 1998 at our center. The protocol was reviewed by the Institutional Review Board of the University of Pittsburgh and informed consent was obtained from all patients.

Patient selection. Criteria for inclusion in this study were as follows: (1) multivessel CAD (>70\% stenosis) involving the LAD distribution with Canadian Cardiovascular Society angina class III or greater; (2) LAD lesion not ideal for per-
Table II. Patient characteristics $(n=31)$

\begin{tabular}{lc}
\hline Age (y) & $69(46-86)$ \\
Age $>75$ y & $13(42 \%)$ \\
Male & $22(71 \%)$ \\
Left ventricular ejection fraction (\%) & $46.3 \pm 12$ \\
Left ventricular ejection fraction $<35 \%$ & $6(19 \%)$ \\
Unstable angina & $23(74 \%)$ \\
Recent (<48 h) myocardial infarction & $5(16 \%)$ \\
Urgent revascularization & $5(16 \%)$ \\
Parsonnet score > 20\% & $8(26 \%)$ \\
Chronic obstructive pulmonary disease $(\%)$ & $18(58 \%)$ \\
Renal dysfunction & $6(19 \%)$ \\
Previous cerebrovascular accident & $17(55 \%)$ \\
Severe peripheral vascular disease & $10(32 \%)$ \\
Morbid obesity & $11(35 \%)$ \\
Non-insulin-dependent diabetes mellitus & $8(25 \%)$ \\
Insulin-dependent diabetes mellitus & 0 \\
Intra-aortic balloon pumping & $2(6 \%)$ \\
Congestive heart failure & $4(13 \%)$ \\
Extent of CAD: & \\
One-vessel CAD & $2(7 \%)$ \\
Two-vessel CAD & $15(48 \%)$ \\
Three-vessel CAD & $8(26 \%)$ \\
Left main CAD & $6(19 \%)$ \\
\hline
\end{tabular}

$C A D$, Coronary artery disease.

cutaneous coronary intervention (type C American Heart Association/American College of Cardiology classification ${ }^{7}$; (3) circumflex and/or right coronary artery lesions suitable for percutaneous coronary intervention (type A or B). Patients were excluded if there was need for associated procedures (eg, carotid or valve disease).

In 1997 (January to December) our center performed 891 surgical coronary revascularizations, including 120 MICABGs. The relatively small number of patients found eligible for inclusion in this study (31 patients over 15 months from September 1996 to January 1998) can be explained by the low level of awareness for integrated coronary revascularization by our referring cardiologists and by the absence of a systematic screening for eligible patients.

Patient characteristics are listed in Table II.

Revascularization strategy. Every candidate for integrated coronary revascularization was discussed by an interdisciplinary team composed of the referring cardiologist, an interventional cardiologist (H.A.C.), and a cardiac surgeon with experience in MICABG (M.Z. or B.P.G.). The procedure committee determined the revascularization strategy on the basis of the angiographic extent of $\mathrm{CAD}$, patient preferences, and alternative options. A decision was reached by group consensus. In case of left main CAD, MICABG was always performed first, to protect the left main coronary artery for the percutaneous coronary intervention ${ }^{8}$; for the other patients, the particular procedural sequence was selected on the basis of logistic considerations (catheterization laboratory and operating room schedule). When possible, integrated coronary revascularization was performed during the same day.

Study end points. End points were considered death, 
myocardial infarction, residual angina, need for repeat revascularization, and LITA patency.

Surgical technique. MICABG was performed through a 3inch left minithoracotomy in the fourth or fifth intercostal space under direct vision of the surgeon, without removal of any costal cartilage or rib. Intravenous infusion of diltiazem (3-5 $\mathrm{mg} / \mathrm{h}$ ), as an ischemic preconditioning agent, was used in all patients. The LITA was harvested circumferentially as a pedicle for its entire length from the subclavian artery takeoff to the bifurcation (average length of LITA pedicle $=15$ $\mathrm{cm}$ ). A dedicated LITA retractor (CardioThoracic Systems Inc, Cupertino, Calif) was successfully used and provided excellent exposure of the entire bed of the LITA. The heparin dose used was $100 \mathrm{IU} / \mathrm{kg}$. The LITA-LAD anastomosis was performed with the use of a mechanical stabilizer (CardioThoracic Systems Inc) without pharmacologic bradycardia. The LITA anastomosis was performed with the use of two separate 8-0 polypropylene sutures at the heel and toe ("double parachute" technique). Before final tying of the suture, the anastomosis was probed with a nonocclusive 1$\mathrm{mm}$ Parsonnet probe in three directions: proximal LAD, distal LAD, and proximal LITA; furthermore, the LITA was flushed through the open suture line before final tying of the knot to eliminate potential thrombus at the site of application of the vascular occluder. On completion of the anastomosis, the flow pattern of the LITA was analyzed intraoperatively with a customized ultrasound transit-time flowmeter (Transonic Systems Inc, Ithaca, NY) as previously described. ${ }^{9}$ Extubation was attempted in all cases in the operating room. Postoperative pain control was achieved by a preoperatively placed epidural catheter or by intercostal nerve block when an epidural catheter was contraindicated (eg, with intravenous heparin infusion). Aspirin (325 mg once a day) was started within 6 hours of MICABG. If percutaneous coronary intervention included the placement of a stent, patients also received ticlopidine ( $250 \mathrm{mg}$ twice daily).

Angiographic confirmation of LITA-LAD anastomotic patency was performed during the primary hospitalization. Follow-up protocol included exercise or pharmacologic stress thallium myocardial scintigraphy at 1 month and Doppler echocardiography of LITA flow ${ }^{10}$ and physical examination at 1,6 , and 12 months' follow-up.

Definitions. The goal of integrated coronary revascularization was revascularization of all viable myocardial territories served by one of the three major coronary arteries (LAD, circumflex artery, right coronary artery) with a coronary stenosis greater than $70 \%$ of the reference diameter and a target vessel with a diameter greater than $1.5 \mathrm{~mm}$. Patients were divided in two groups: group A included patients who received an anatomically complete revascularization and group B those with an anatomically incomplete but functionally adequate revascularization (ie, revascularization of nonviable myocardium or areas served by a target vessel $<1.5$ $\mathrm{mm}$ of diameter was not performed). ${ }^{11}$

Statistical analysis. Data were expressed as mean \pm standard deviation. Group comparisons were performed by means of Fisher's exact test.

\section{Results}

Integrated coronary revascularization was performed in all 31 patients without mortality. During MICABG, LITA was anastomosed to the LAD in 28 patients, to the diagonal branch of the LAD in 2 patients (because of a diffusely diseased LAD in 1 patient and intramyocardial LAD in the other), and to both the LAD and diagonal as a Y graft using the left radial artery in 1 patient. The time to complete the LITA anastomosis was $14.6 \pm 5.2$ minutes and the operating time (skin to skin) was $105 \pm 20$ minutes; 28 patients were extubated in the operating room $(28 / 31=90 \%)$ and the remaining 3 were extubated within 2 hours. No patient required re-exploration for bleeding or conversion to sternotomy. There were no perioperative myocardial infarctions. Two patients with left main coronary artery stenosis had an intra-aortic balloon pump already in place at the time of MICABG. One patient had undergone a previous left lower lobectomy. One patient had undergone previous CABG with occluded vein grafts. One patient had idiopathic thrombocytopenia (platelet count $15,000 / \mathrm{mm}^{2}$ ) but did not require platelet transfusion during or after MICABG.

Only 1 patient had atrial fibrillation after the operation $(1 / 31=3 \%)$. Two patients $(2 / 31=6.4 \%)$ required transfusion of packed red blood cells. LITA anastomosis was confirmed to be patent by angiography (26 patients) or intraoperative LITA flow pattern analysis ${ }^{9}$ (5 patients) in all patients (100\%; 95\% confidence limits $=0.89-1)$. In both patients in whom the LITA was anastomosed to the diagonal branch, the LAD was retrogradely filled without restriction by the LITA at follow-up angiography.

Details of percutaneous coronary intervention are listed in Table III. Percutaneous coronary intervention was performed before MICABG (1 day before in 1 case and 2 days before in the other) in 2 patients (7\%), on the same day of MICABG in 16 patients (52\%), on postoperative day 1 in 3 patients (9\%), and on postoperative days 2 to 4 in 10 patients (32\%). In 1 patient who required abciximab during stent placement, MICABG was performed 2 days later to avoid bleeding complications.

Overall, integrated coronary revascularization allowed a revascularized vessel/patient ratio of 2.26. Postprocedure length of stay in the hospital was $2.7 \pm 1.0$ days, and 13 patients (42\%) were discharged home on postoperative day 1 or 2 .

The degree of revascularization achieved was anatomically complete in 21 patients $(21 / 31=68 \%$; group A) and anatomically incomplete but functionally adequate in 10 patients $(10 / 31=32 \%$; group $\mathrm{B})$. 
Table III. Percutaneous coronary interventions

\begin{tabular}{lc}
\hline & Patients (\%) \\
\hline Target vessels & \\
RCA & $9(29 \%)$ \\
CX & $9(29 \%)$ \\
OM & $9(29 \%)$ \\
LMCA & $4(13 \%)$ \\
Diagonal & $3(10 \%)$ \\
Ramus & $2(6 \%)$ \\
Posterolateral branch of RCA & $2(6 \%)$ \\
Interventricular posterior coronary artery & $1(3 \%)$ \\
Extent of percutaneous coronary intervention & \\
Single vessel & $24(77 \%)$ \\
Double vessel & $6(19 \%)$ \\
Triple vessel & $1(3 \%)$ \\
Type of percutaneous coronary intervention & \\
Elective stent & $23(60 \%)$ \\
"Plain old balloon angioplasty" & $13(34 \%)$ \\
Rotational atherectomy + PTCA & $2(6 \%)$ \\
\hline
\end{tabular}

PTCA, Percutaneous transluminal coronary angioplasty; $R C A$, right coronary artery; Ramus, ramus intermedius; $O M$, obtuse marginal; $C X$, circumflex; $L M C A$, left main coronary artery.

Anatomically complete revascularization was achieved more frequently in patients with lower preoperative risk profile (Parsonnet score $\left.{ }^{12}<20 \%\right)(18 / 23=78 \%)$ than in patients with higher risk (Parsonnet $>20 \%)(3 / 8=$ $37.5 \%)(P=.07$; Fisher's exact test, 2-tailed).

Three patients $(9.6 \%)$ required repeat target vessel revascularization. One patient who underwent MICABG and stent of the obtuse marginal branch of the circumflex coronary artery had a subacute thrombosis of the obtuse marginal stent 4 days after the procedure because of noncompliance with the aspirin/ticlopidine regimen. She underwent percutaneous transluminal coronary angioplasty (PTCA) of the thrombosed stent that eventually restenosed requiring single-vessel CABG to the obtuse marginal branch; at operation, the LITA-LAD anastomosis was widely patent. Another patient had new critical disease proximal to a nonstenotic right coronary artery stent 3 months after integrated coronary revascularization; he underwent new stent placement of the proximal right coronary artery without complications. The third patient had new significant disease develop proximal to a stent of the distal obtuse marginal branch in a diffusely diseased vessel 6 months after integrated coronary revascularization and required deployment of 2 additional stents to the proximal circumflex artery. One of these patients requiring repeat revascularization was in group A (anatomically complete revascularization) $(1 / 21=5 \%)$ and 2 patients were in group B (incomplete but functionally adequate revascularization $)(2 / 10=20 \%)$.
Table IV. Results (mean follow-up $=10.8 \mathrm{mo}$ )

\begin{tabular}{ll}
\hline Survival & $100 \%(31 / 31)[C L=0.89-1]$ \\
Freedom from angina/MI* & $100 \%(31 / 31)[C L=0.89-1]$ \\
LITA patency & $100 \%(31 / 31)[C L=0.89-1]$ \\
Freedom from repeat & $90.4 \%(28 / 31)[C L=0.74-0.98]$ \\
$\quad$ revascularization &
\end{tabular}

$\overline{C L, 95 \% \text { Confidence limits; LITA, left internal thoracic artery; } M I \text {, myocardial }}$ infarction.

*After repeat revascularization in 3 patients.

Excluding the 3 patients requiring repeat revascularization, adenosine stress thallium myocardial scintigraphy was performed in 21 patients $(21 / 28=75 \%)$ and was negative for the presence of reversible ischemic defects. Noninvasive Doppler assessment of LITA ${ }^{10}$ demonstrated maintained patency of the anastomosis up to 10.8 months of follow-up in all patients.

Results are summarized in Table IV.

\section{Discussion}

The last few years have witnessed a significant expansion of the therapeutic options available for patients with CAD requiring myocardial revascularization. Meta-analysis of recent major trials have shown that PTCA and CABG are associated with no difference in major adverse cardiac events. ${ }^{13}$ However, PTCA is associated with a higher need for repeat revascularization caused by restenosis. ${ }^{14}$ Furthermore, there is evidence from randomized studies that restenosis is higher in the LAD than in non-LAD lesions. Patients enrolled in the CAVEAT (Coronary Angioplasty Versus Excisional Atherectomy Trial) study ${ }^{15}$ with proximal LAD stenosis had a $63 \%$ restenosis rate after PTCA, compared with a $50 \%$ restenosis rate in non-LAD lesions. In the Stent ReStenosis Study (STRESS), ${ }^{16}$ in which LAD lesion location was compared with nonLAD lesion location at the time of intervention, the vessels in the LAD group were smaller $(2.85 \pm 0.4 \mathrm{~mm}$ vs $3.09 \pm 0.4 \mathrm{~mm} ; P<.001)$, with less acute coronary lumen gain after intervention $(1.3 \pm 0.49 \mathrm{~mm}$ vs $1.57 \pm$ $0.5 \mathrm{~mm} ; P<.001)$, which translated into a smaller mean luminal diameter at follow-up $(1.46 \pm 0.6 \mathrm{~mm}$ vs $1.76 \pm 0.6 \mathrm{~mm} ; P<.001)$, and a higher diameter stenosis $(48.85 \% \pm 19.03 \%$ vs $42.9 \% \pm 18.7 \% ; P<.001)$. The target vessel revascularization rate of patients with LAD lesions treated was $19 \%$ versus $11 \%$ in the nonLAD lesion location. In the PTCA group, the lesions in the LAD location had a binary restenosis rate of $62 \%$ compared with $32 \%$ in the non-LAD location. The introduction of stents has decreased overall restenosis rate to $31.6 \%$ in STRESS and $22 \%$ in the Belgium 
Netherlands Stent Study Group (BENESTENT ), ${ }^{17}$ but recent data from the Washington Hospital Center show that LAD location is still a major factor affecting loss index after stenting (M. Leon, personal communication, September 1998).

The LAD is a strategically important artery, and the patency of the LAD is an important determinant of survival because of the large territory of myocardium supplied (up to $70 \%$ of the left ventricle). ${ }^{18}$ Revascularization of the LAD with the LITA is associated with improved survival $(86.6 \%$ vs $75.9 \%$ actuarial survival at 10 years; $P<.001$ ), freedom from cardiac events, and long-term graft patency compared with CABG with only saphenous vein grafts. ${ }^{19}$ The favorable effects on mortality and morbidity are observed irrespective of age, gender, or left ventricular function. ${ }^{20}$ Prospective studies comparing CABG and medical therapy have suggested that revascularization does not necessarily improve survival in patients with single- or double-vessel CAD unless one of the vessels is the proximal LAD. ${ }^{21}$ Presence of atherosclerotic lesions in non-LAD vessels affects the event rates of patients with vein grafts but not of patients with LITA grafts. ${ }^{20}$ Cameron and associates ${ }^{22}$ observed that a durable arterial graft to the LAD is a more powerful predictor of survival than is progressive coronary atherosclerosis in other coronary arteries. A unique feature of LITA used as a bypass conduit is a striking resistance to atheroma, with 10 years' patency well above $90 \%$. The LITA is also able to dilate in vivo, ${ }^{23}$ physiologically adapting to the flow requirement of the myocardium.

CABG with the LITA is clearly the revascularization modality of choice for the LAD. With the introduction of MICABG, the prognostic benefits of surgery may now be gained without the drawbacks of sternotomies, leg wounds, and cardiopulmonary bypass. We have demonstrated that MICABG reduces mortality, cerebral events, and length of stay in extremely high-risk patients. ${ }^{24}$ MICABG was also found to be associated with significantly decreased need for repeat revascularization compared with stenting for type $\mathrm{C}$ lesions of the LAD. ${ }^{25}$ The major limitation of MICABG appears to be the inaccessibility of the circumflex and right coronary arteries. Standard CABG with saphenous vein grafts to these coronary vessels is associated with a patency of $60 \%$ at 10 years and freedom from saphenous vein graft disease in only $50 \%$ of grafts. ${ }^{26}$

In an attempt to offer the best revascularization strategy to patients with multivessel CAD, we hypothesized that integrated coronary revascularization, by combining MICABG of the LAD with the LITA and percutaneous coronary intervention with predominant use of stents, could provide "the best of two possible worlds," promoting a complementary role of minimally invasive surgery and interventional cardiology.

In our series of 31 consecutive patients undergoing interventional coronary revascularization, the acute procedural success rate was $100 \%$. Freedom from repeat revascularization at 10.8 months' follow-up is $90.4 \%$; one case of subacute thrombosis owing to noncompliance with aspirin/ticlopidine regimen occurred, and 2 cases of recurrence of CAD proximal to the site of stent placement were noted. No clinical restenosis was observed. Patency of the LITA-LAD anastomosis was $100 \%$. Survival is $100 \%$ at 10.8 months. Unlike Angelini and colleagues, ${ }^{5}$ we did not observe increased bleeding caused by the more intense anticoagulation required for stents.

In conclusion, on the basis of these early results, we believe that integrated coronary revascularization is feasible and safe. Since conventional CABG represents a gold standard against which any new approaches to coronary revascularization need to be tested, we have designed the MORE trial; this is a prospective randomized trial comparing integrated coronary revascularization and $\mathrm{CABG}$ for suitable patients with multivessel CAD involving the LAD.

We agree with a recent editorial of Simoons ${ }^{27}$ that it is reasonable for the future to envision the formation of a practical "partnership" between interventional cardiology and coronary bypass surgery, with the development of revascularization centers in which different procedures are performed by a closely coordinated team of operators with different backgrounds.

\section{REFERENCES}

1. Doty JR, Fonger JD, Nicholson CF, Sussman MS, Salomon NW. Cost analysis of current therapies for limited coronary revascularization. Circulation 1997;96(Suppl):II16-20.

2. King RC, Reed TB, Hurst JL, Shockey KS, Tribble CG, Spotnitz WD, et al. Minimally invasive coronary artery bypass grafting decreases hospital cost and stay. Ann Surg 1997;225:805-11.

3. Zenati M, Domit TM, Saul M, Gorcsan J, Katz WE, Hudson M, et al. Resource utilization for minimally invasive direct and standard coronary artery bypass. Ann Thorac Surg 1997;63:S84-7.

4. Calafiore AM, Di Giammarco G, Teodori G, Gallina S, Maddestra N, Paloscia L, et al. Midterm results after minimally invasive coronary surgery (LAST operation). J Thorac Cardiovasc Surg 1998;115:763-71.

5. Angelini GD, Wilde P, Salerno TA, Bosco G, Calafiore AM. Integrated left small thoracotomy and angioplasty for multivessel coronary artery revascularization [letter]. Lancet 1996;347:757-8.

6. Friedrich GJ, Bonatti J, Dapunt OE. Preliminary experience with minimally invasive coronary artery bypass surgery combined with coronary angioplasty [letter]. N Engl J Med 1997;336:1454.

7. Ryan TJ, Bauman WB, Kennedy JW, Keriakes DJ, King SB, 
McCallister BD, et al. Guidelines for percutaneous transluminal coronary angioplasty. Circulation 1988;88:2987-3003 .

8. Zenati M, Cohen HA, Chough SH, Smith CA, Lee JS, Feldman $\mathrm{M}$, et al. Integrated coronary revascularization for left main coronary disease: an alternative approach with minimally invasive bypass surgery and TCT [abstract]. Am J Cardiol 1997;80:(Suppl I): 85.

9. Mandarino WA, Gorcsan J, Katz WE, Cohen HA, Griffith BP, Zenati M. Intraoperative analysis of left internal mammary artery graft flow patterns following minimally invasive coronary bypass [abstract]. J Am Coll Cardiol 1998;31:464A-5A.

10. Katz WE, Zenati M, Gorcsan J. Noninvasive assessment of the mammary artery graft after minimally invasive coronary bypass using adenosine Doppler echocardiography [abstract]. J Am Coll Cardiol 1998;31:386A.

11. Faxon DP, Ghalilli K, Jacobs AK, Ruocco NA, Christellis EM, Kellett MA, et al. The degree of revascularization and outcome after multivessel coronary angioplasty. Am Heart J 1993;123: 854-9.

12. Parsonnet V, Dean D, Bernstein AD. A method of uniform stratification of risk for evaluating the results of surgery in acquired adult heart disease. Circulation 1989;79(Suppl):I3-12 .

13. Pocock SJ, Henderson RA, Rickards AF, Hampton JR, King SB, Hamm CW, et al. Meta-analysis of randomized trials comparing coronary angioplasty with bypass surgery. Lancet 1995;346: $1184-9$.

14. Popma JJ, Topol EJ. Factors influencing restenosis after coronary angioplasty. Am J Med 1990;88:1-16.

15. Elliott JM, Berdan LG, Holmes DR, Isner JM, King SB, Keeler $\mathrm{GP}$, et al. One-year follow-up in the coronary angioplasty versus excisional atherectomy trial (CAVEAT I). Circulation 1995;91: 2158-66.

16. Fishman DL, Leon MB, Baim DS, Schatz RA, Savage MP, Penn I, et al. A randomized comparison of coronary stent placement and balloon angioplasty in treatment of coronary artery disease. N Engl J Med 1994;331:496-501.

17. Serruys PW, Emanuelsson H, Van Der Giessen W, et al. Heparincoated Palmatz-Schatz stent in human coronary arteries: early outcome of the Benestent-II pilot study. Circulation 1996;93:41222.

18. Mahmarian JJ, Pratt CM, Boyce TM, Verani MS. The variable extent of jeopardized myocardium in patients with single vessel coronary artery disease: quantification by thallium-201 single photon emission computed tomography. J Am Coll Cardiol 1991; 17:355-62.

19. Loop FD, Lytle BW, Cosgrove DM, Stewart RW, Goormastic M, Williams GW, et al. Influence of the internal mammary artery graft on 10 year survival and other cardiac events. N Engl J Med 1986;314:1-6.

20. Loop FD. Internal thoracic artery grafts: biologically better coronary arteries. N Engl J Med 1996;334:263-5.

21. Coronary Artery Surgery Study Principal Investigators. Myocardial infarction and mortality in the CASS randomized trial. N Engl J Med 1984;310:750-8.

22. Cameron A, Davis KB, Green G, Schaff HV. Coronary bypass surgery with internal thoracic artery grafts: effect on survival over a 15-year period. N Engl J Med 1996;334:216-9.

23. Hanet C, Schroeder E, Michel X, et al. Flow-induced vasomotor response to tachycardia of the human internal mammary artery and saphenous vein grafts late following bypass surgery. Circulation 1991;84(Suppl);III268-74.
24. Zenati M, Cohen HA, Holubkov R, Smith AJ, Boujoukos A, Caldwell J, et al. Preoperative risk models for minimally invasive coronary bypass: a preliminary study. J Thorac Cardiovasc Surg 1998;116;584-9.

25. Mariani MA, Boonstra PW, Grandjean JG, Peels JOJ, Monnink SHJ, Hejier PD, et al. Minimally invasive coronary artery bypass grafting versus coronary angioplasty for isolated type $\mathrm{C}$ stenosis of the left anterior descending artery. J Thorac Cardiovasc Surg 1997;114:434-9.

26. Motwani JG, Topol EJ. Aortocoronary saphenous vein graft disease: pathogenesis, predisposition and prevention. Circulation 1998;97:916-31.

27. Simoons ML. Myocardial revascularization-Bypass surgery or angioplasty? [editorial]. N Engl J Med 1996;335:275-7.

\section{Discussion}

Dr Steven W. Guyton (Seattle, Wash). It is important for all of us to realize that there will be pressure from our cardiologist colleagues to do this type of hybrid procedure, or perhaps I should call it a "bastardized" procedure. In truth, many such procedures will be done. It is important that this type of procedure be studied, and I welcome having surgeons involved in studying them.

You report on 31 consecutive patients, who actually should be called 31 highly selected patients: they had $70 \%$ stenosis of the LAD and other coronary arteries, class III or IV angina or what was termed objective evidence of ischemia, their nonLAD lesions were type A or B for percutaneous coronary intervention, and they had no associated procedures. Fifty-five percent of these patients had prior strokes; $35 \%$ of them were morbidly obese. Every candidate was discussed by an interdisciplinary team, and I am encouraged that a surgeon was included in that interdisciplinary team. How many patients were discussed by this team to arrive at these 31 patients?

Dr Zenati. These 31 patients were the only ones who fulfilled the inclusion criteria we set at the beginning of the study. Early on, the awareness of this option was not widespread among our cardiologists, and we did not systematically screen patients for eligibility in this study.

Dr Guyton. How many coronary bypasses and angioplasties were done during that same period?

Dr Zenati. In a 12-month period, we usually do about 900 CABGs.

Dr Guyton. Would you say that 5\% of patients are appropriate for this sort of procedure?

Dr Zenati. I would think that it would be a much larger percentage. A lot of these patients currently have multivessel stenting instead of integrated revascularization.

Dr Guyton. You talk about multivessel disease, but in percutaneous interventions only a single vessel was done in $97 \%$ of the patients. The total number of revascularizations done in these 31 patients was 64, I believe, for an average number of 2 revascularizations per patient.

Dr Zenati. The average number of revascularizations per patient is actually 2.3 .

Dr Guyton. Essentially, then, you are treating double-vessel disease. 
Dr Zenati. Most of our patients had double-vessel disease, but a quarter had triple-vessel disease.

Dr Guyton. Integrated coronary revascularization seems to me to be a convenient way to achieve complete revascularization in patients who refuse to consider a sternotomy or in patients in whom the surgeons consider a sternotomy to be too dangerous. Alternatively, integrated coronary revascularization gives "functionally adequate" results in patients with more diffuse disease who again are considered too high a risk for $\mathrm{CABG}$.

Diabetes is not mentioned in the paper. Did any of these patients have diabetes?

Dr Zenati. About 25\% had diabetes, but none of them had insulin-dependent diabetes.

Dr Guyton. One of the things you might want to consider in your randomized study is excluding patients with diabetes, because of the long-term poor results with angioplasty.

Dr Zenati. Yes, you are referring to the results of the Bypass vs. Angioplasty Revascularization Investigation trial, and you are probably aware of the controversy concerning the influence of diabetes on results of percutaneous intervention. That is the reason that the BARI II trial was designed.

Dr Guyton. It certainly may be a confounding factor, though, in looking at your randomized trial. You point out the narrowing gap between percutaneous intervention and surgical revascularization in terms of invasiveness and cost. Outcome data, however, are comparable only in fairly shortterm results. Your goal of revascularization brings out a fairly disturbing concept of "functionally adequate," in which you are revascularizing down to vessels at $1.5 \mathrm{~mm}$ in areas where you can demonstrate ischemia. This was stated as the only goal of your randomized trial when I looked up the protocol on the Internet. You talked in your paper about the best possible short- and long-term results; yet the maximum results in your paper are about 20 months, with average results at 10 months. Your myocardial optimal revascularization trial proposes only a 1-year follow-up, which I find very disturbing. Also, no end points are defined that would measure late revascularization failures.

In this era, as the moving target of surgical revascularization is coming to arterial grafts with improved results in a second decade after CABG, I think it is important to compare the best available hybrid procedure that you want to recommend or want to study to the best available surgical option, that of arterial revascularization.

Dr Zenati. I appreciate your comments, and certainly we are aware of the importance of long-term results in a revascularization study. The trial that we are setting up is based on the funding we had available, which allows a 1-year followup, but that is not saying that a longer follow-up would not be necessary. Regarding the controversy between "anatomically complete" and "functionally adequate," I would like to stress that our approach is not different from what we do every day in the operating room. If we have a patient with a previous transmural inferior myocardial infarction and occluded right coronary artery, we do not feel guilty in not bypassing the posterior descending artery; although in this setting we are not performing complete revascularization, we believe that it is functionally adequate for the patient.

Dr Guyton. Long-term results are best in patients whom we consider to be completely revascularized. I find it disturbing, therefore, that you state that your goal is to achieve "functionally adequate" revascularization rather than complete revascularization.

Dr Zenati. I agree with you that revascularization should be as complete as possible, but I think the whole issue of completeness of revascularization is a matter of definition.

Dr Vaughn A Starnes (Los Angeles, Calif). I too think this study needs to be done. We know the operation is no better than its weakest link. What discussions go on before placing a patient in this protocol in terms of if it is a vessel that can undergo PTCA and stent with reliable results? As we see your short-term results, we have 3 patients coming to re-intervention, 1 patient with $\mathrm{CABG}$ with a vein graft, which I assume was done on bypass.

Dr Zenati. Yes.

Dr Starnes. Two more came back for re-intervention with repeat stenting, and history tells us that those procedures will likely fail too and require a repeat bypass operation. What goes into the team evaluation of a particular lesion in terms of whether the vessel can be opened reliably with PTCA and get acceptable long-term results? Long-term results and multiple stents in a single artery would argue against a long-term satisfactory outcome. These patients, rather than being randomized, should have CABG.

Dr Zenati. I agree. In fact, we excluded patients with type $\mathrm{C}$ lesions from percutaneous intervention. We think that for patients with type A or B lesions percutaneous intervention yields very good results. We do not think the integrated approach will be replacing any existing alternative revascularization available, but we think it will have a role for selected patients, specifically the $\mathrm{C}$ lesions of LAD and type A/B lesions of non-LAD vessels.

Dr Steven R. Gundry (Loma Linda, Calif). I appreciate your bringing this hybrid procedure to our memberships' attention, but I have a real problem with taking essentially two inferior procedures, combining them, and promoting them as a better procedure. I have a problem with saying that you have $100 \%$ patency of the LAD anastomosis at 1 day or at 1 hour, projecting that, and saying that at 1 year the anastomoses are still patent. In our study of off-bypass patients, it actually took approximately $2 \frac{1}{2}$ years for the problems with the anastomoses to surface. In fact, all of our patients had negative stress tests after the operation, proving that the anastomoses were patent at that time. To claim in your article that you have $100 \%$ patency at 1 year's follow-up when your angiogram was done at 1 day is deceptive. Second, to say that you have $100 \%$ angina-free patients at 1 year, and yet $10 \%$ of them have had to have a re-intervention, is also a bit deceptive.

Can you tell us how many patients have returned to any form of anti-anginal therapy during that 1 -year period? 
Dr Zenati. To answer the first question about the angiography, the control studies were done early, but we also do a follow-up study of flow pattern using Doppler echocardiography. In all of those patients, patency was confirmed at follow-up (maximum 10 months).

About $30 \%$ of patients are receiving calcium channel blockers if they have a history of hypertension.

Dr Gundry. Thank you. I do not think this discussion would be complete without addressing cost, because we must take into account the cost of multiple stents and redo stents. A $10 \%$ failure rate is considered to be a failure of the approach, which must be accounted for, and the cost of the operating room and the surgical approaches must be considered, as well.

Have you analyzed the cost of this approach as an entity in these 30 patients?

Dr Zenati. That is one of the main end points that will be addressed by the trial. There are also several ways one could think of optimizing costs, such as single operating room and single cross-trained nurses. We currently do the procedures the same day in two different rooms, and this does not really alter the schedule too much. However, as you pointed out correctly, cost is going to be an issue and we are very interested in evaluating that. 